\title{
The cDNA Structures and Expression Profile of the Ascorbate Peroxidase Gene Family During Drought Stress in Wild Watermelon
}

\author{
Goitseone Malambane ${ }^{1,2}$, Hisashi Tsujimoto ${ }^{1,3}$ \& Kinya Akashi ${ }^{1,3}$ \\ ${ }^{1}$ United Graduate School of Agricultural Science, Tottori University, Tottori, Japan \\ ${ }^{2}$ Department of Crop Science and Production, Botswana University of Agriculture and Natural Resources, \\ Gaborone, Botswana \\ ${ }^{3}$ Arid Land Research Center, Tottori University, Tottori, Japan \\ Correspondence: Kinya Akashi, United Graduate School of Agricultural Science, Tottori University, 4-101, \\ Koyama-Minami, Tottori 680-8553, Japan. Tel: 81-857-31-5352. E-mail: akashi.kinya@tottori-u.ac.jp
}

Received: April 20, 2018

Accepted: June 2, $2018 \quad$ Online Published: July 15, 2018

doi:10.5539/jas.v10n8p56

URL: https://doi.org/10.5539/jas.v10n8p56

\begin{abstract}
Ascorbate peroxidase (APX) plays an important role in detoxifying reactive oxygen species under environmental stress. Although previous work in drought-tolerant wild watermelon has shown an increase in chloroplast APX enzyme activity under drought, molecular entities of APX have remained uncharacterized. In this study, structure and transcriptional regulation of the APX gene family in watermelon were characterized. Five APX genes, designated as CLAPX1 to CLAPX5, were identified from watermelon genome. The mRNA alternative splicing was suggested for $C L A P X 5$, which generated two distinct deduced amino acid sequences at their C-terminus, in resemblance to a reported alternative splicing of chloroplast APXs in pumpkin. This observation suggests that two isoenzymes for stromal and thylakoid-bound APXs may be generated from the CLAPX5 gene. Phylogenetic analysis classified CLAPX isoenzymes into three clades, i.e., chloroplast, microbody, and cytosolic. Physiological analyses of wild watermelon under drought showed a decline in stomatal conductance and $\mathrm{CO}_{2}$ assimilation rate, and a significant increase in the enzyme activities of both chloroplast and cytosolic APXs. Profiles of mRNA abundance during drought were markedly different among CLAPX genes, suggesting distinct transcriptional regulation for the APX isoenzymes. Up-regulation of CLAPX5-I and CLAPX5-II was observed at the early phase of drought stress, which was temporally correlated with the observed increase in chloroplast APX enzyme activity, suggesting that transcriptional up-regulation of the CLAPX5 gene may contribute to the fortification of chloroplast APX activity under drought. Our study has provided an insight into the functional significance of the $C L A P X$ gene family in the drought tolerance mechanism in this plant.
\end{abstract}

Keywords: alternative splicing, ascorbate peroxidase, drought stress, ROS, wild watermelon

\section{Introduction}

Drought-associated water deficit is one of the major factors restricting plant productivity and crop yields worldwide. To sustain themselves when exposed to water deficit, plants activate various physiological and metabolic mechanisms that protect them from adverse physicochemical injuries. Some of the key molecules generated in large quantities in plant cells during environmental stress are the reactive oxygen species (ROS), such as hydrogen peroxide $\left(\mathrm{H}_{2} \mathrm{O}_{2}\right)$, superoxide radical $\left(\mathrm{O}_{2}{ }^{-}\right)$, and hydroxyl radical $(\cdot \mathrm{OH})(\mathrm{Gill}$ \& Tuteja, 2010; You \& Chan, 2015). These species oxidize various cellular components, such as nucleic acids, lipids, and proteins, which can cause lethal damage to plants. Plants are equipped with several enzymatic and non-enzymatic systems that decompose these ROS and maintain them at nontoxic levels (Mittler et al., 2004). The enzymes that scavenge ROS include superoxide dismutase (SOD), catalase (CAT), glutathione peroxidase (GPX), and ascorbate peroxidase (APX). Moreover, an array of non-enzymatic antioxidants, such as ascorbate, glutathione, carotenoids, and tocopherols, serve as defense agents for protecting plant cells from oxidative injuries. These enzymes and antioxidants are mutually related in the glutathione-ascorbate cycle, which plays an essential role in resisting water deficit and oxidative stress under drought (Li et al., 2013).

The importance of studying wild plant species, in helping to solve problems of disease and pest resistance, and to improve yields and adaptation to extreme environments, has been widely emphasized (Hawkes, 1991; Henry \& 
Nevo, 2014; Brozynska et al., 2016). One of the most drought-tolerant wild plant species is wild watermelon (Citrullus lanatus), which inhabits very harsh conditions in the Kalahari Desert and has the capacity to thrive under high light and drought stress (Akashi et al., 2008; Yoshimura et al., 2008). Although the fruit of this plant is not palatable to humans, it is a very important source of water for wildlife inhabitants in the desert, especially during long dry spells. Unraveling the mechanisms responsible for drought tolerance in the wild watermelon will offer valuable insights for breeding other crops, which are currently susceptible to adverse drought effects, toward improved drought tolerance.

A previous study showed that the enzyme activity of chloroplast APX increased in the leaves of wild watermelon under drought stress (Nanasato et al., 2010). APX catalyzes the conversion of $\mathrm{H}_{2} \mathrm{O}_{2}$ to water with concomitant oxidation of ascorbate to monodehydroascorbate, thereby playing a pivotal role in the detoxification of ROS under biotic or abiotic stresses (Asada, 2006; Miyake et al., 2006). Extensive research has shown that APX isoenzymes are localized in at least three different subcellular compartments in plant cells, namely, cytosol (cAPX), microbody (mAPX), and chloroplast (Ishikawa \& Shigeoka, 2008). The chloroplastic APXs are further categorized into at least two isoenzymes according to their distinct microenvironments, i.e. stroma-soluble (sAPX) and thylakoid membrane-bound (tAPX) forms. Interestingly, plants appear to be divided into two groups according to their mode of biogenesis of sAPX and tAPX. In the first plant group (which includes Arabidopsis, rice, and tomato), sAPX and tAPX are encoded by distinct genes. In the second plant group (which includes spinach, tobacco, and pumpkin), on the other hand, sAPX and tAPX are encoded by a single gene, and their protein products are generated by post-transcriptional alternative splicing of the mRNA precursors (Ishikawa \& Shigeoka, 2008).

Another feature of APX enzymes is their instability in the absence of ascorbate (Hiner et al., 2000; Kitajima et al., 2006). APX isoenzymes in chloroplasts are particularly sensitive to inactivation under ascorbate-deficient conditions, in comparison to their cAPX and mAPX counterparts (Yoshimura et al., 1998). Although increased expression of cAPX under various abiotic stresses is well documented in plants (Karpinski et al., 1997; Shigeoka et al., 2002), reports on the up-regulation of chloroplast APXs under drought and high light stress have been limited, and even a decrease in chloroplast APX activity was reported in spinach under high light stress (Yoshimura et al., 2000). These observations suggest that chloroplast APX may be one of the initial targets for oxidative injuries in plant leaves under drought- and light-related stress (Shikanai et al., 1998). In this regard, the observed increase in the chloroplast APX activity in wild watermelon under water deficit stress (Nanasato et al., 2010) is intriguing because it may suggest that this plant fortifies the activity of chloroplast APXs that are otherwise vulnerable to drought and high light conditions. However, to our best knowledge, no report has been found on the structure and mRNA expression profiles of the APX gene family in watermelon species. Therefore, in this study, we attempted to characterize the gene organization and transcriptional regulation of the putative APX gene family in wild watermelon, together with the physiological responses of this plant during drought and high light stress conditions.

\section{Method}

\subsection{Plant Materials and Growth Conditions}

Wild watermelon (Citrullus lanatus Acc. No. 101117-1) (Yoshimura et al., 2008) were self-pollinated at least three times and their seeds were used in this study. The seeds were soaked overnight in water at $30{ }^{\circ} \mathrm{C}$ in the dark and planted in pots filled with a horticulture soil the following morning. The germinated seedlings were grown in a growth chamber under LED lights with a light intensity of 800-1,000 $\mu$ mol photons $\mathrm{m}^{-2} \mathrm{~s}^{-1}$ under a $14 \mathrm{~h}$ light and $10 \mathrm{~h}$ dark photoperiod, with an air temperature of $30^{\circ} \mathrm{C}$ and relative humidity of $50 \%$, for the entire growth and monitoring periods. After germination the plants were watered daily with supplementation of a 1,000-fold diluted Hyponex nutrient solution (Hyponex Japan Corp., LTD, Osaka, Japan) twice a week. When they reached the stage when the fifth true leaf had fully expanded, drought stress was introduced by withholding watering.

\subsection{Characterization of Gene and cDNA Structures for Watermelon APXs}

Watermelon cDNA and genomic sequences for the putative APX genes were searched using TBLASTN against a genome sequence database of $C$. lanatus subsp. vulgaris cv. 97103 in the Cucurbit Genomics Database (Guo et al., 2013), with known Arabidopsis APX sequences as the queries, using BLOSUM62 matrix and a setting of gap opening and extension penalties for 11 and 1, respectively, and a threshold E-value of 1e-10. The genome and unigene sequences, and chromosome locations of watermelon APX genes were obtained from the Cucurbit Genomics Database.

For PCR amplification of the whole coding DNA sequences (CDSs) of wild watermelon, a leaf of the watermelon that was exposed to water deficit stress for three days was used for total RNA extraction. The leaf 
sample was snap frozen into liquid nitrogen and stored at $-80^{\circ} \mathrm{C}$ until RNA isolation. Total RNA was extracted with the Spectrum Plant Total RNA Kit (Sigma Aldrich, St Louis, MO, USA), and trace amounts of genomic DNA were degraded using the On-Column DNase I Digestion Set (Sigma Aldrich). The cDNA syntheses were performed using the ReverTra-Ace- $\alpha$ synthesis kit (Toyobo, Osaka, Japan) with an Oligo(dT) primer (Toyobo). To design primers for amplifying whole CDSs, sequences flanking the CDSs, i.e., upstream of the translation start codons and downstream of the stop codons (Table A1 in Appendix), in the unigene information from cv. 97103, were employed. The 3'-RACE was performed according to the instructions from a 3'-Full RACE Core Set (Takara, Shiga, Japan). The PCR amplification was performed by a KOD FX NEO high-fidelity proofreading enzyme (Toyobo). The PCR products were separated in an agarose gel electrophoresis and amplicons were purified from the agarose gel using a MinElute Gel Extraction Kit (QIAGEN, Germantown, MD, USA), and then sub-cloned into an Invitrogen TOPO-BLUNT vector (Life Technologies, Carlsbad, CA, USA). Sequence reactions were performed using a BigDye terminator v3.1 Cycle Sequencing Kit (Life Technology) and analyzed using a 3130xl DNA sequencer (Applied Biosystems, Foster City, CA, USA).

Intracellular locations of these gene products were predicted by the WoLF PSORT and DeepLoc-1.0 programs (Horton et al., 2007; Armenteros et al., 2017). Visualization of the exon-intron structures of the genes was performed by the GSDS 2.0 gene feature visualization server (Hu et al., 2015). ClustalW and ETE3 programs in the GenomeNet server (Kanehisa et al., 2002) were used to generate an amino acid sequence alignment and phylogenetic tree.

\subsection{Measurement of Plant-Water Relations}

Gravimetric soil moisture content was determined essentially as described by Reynolds (1970), with the following minor modification: after harvesting leaf samples, the entire aboveground plant tissues were cut out. The moist soil, together with the planting pot, were then weighed and recorded as the wet mass (WM). The soil was oven dried at $105^{\circ} \mathrm{C}$ for $72 \mathrm{~h}$, then weighed and recorded as the dry mass (DM). The soil moisture content $(\theta \mathrm{g})$ was determined by the following formula (Reynolds, 1970):

$$
\theta \mathrm{g}=(\mathrm{WM}-\mathrm{DM}) / \mathrm{DM} \times 100
$$

Leaf relative water content was measured essentially as described by Barrs (1968), with the following modification: the leaves were harvested and the fresh weight was quickly measured on a Unibloc AUX 120 balance (Shimadzu, Kyoto, Japan) and recorded as the fresh weight (FW). After the FW were recorded, the leaf samples were placed in zip-lock plastic bags, which were filled with distilled water and kept overnight at $25^{\circ} \mathrm{C}$. The next day, excess water was removed by blotting the leaves in paper towels. The water-saturated leaves were then weighed and recorded as turgid weight (TW). The turgid leaves were then oven dried at $80{ }^{\circ} \mathrm{C}$ for $3 \mathrm{~d}$ and their weights were recorded as the dry weights (DW). The leaf relative water content (LRWC) was calculated by the following formula (Barrs, 1968):

$$
\mathrm{LRWC}=(\mathrm{FW}-\mathrm{DW}) /(\mathrm{TW}-\mathrm{DW}) \times 100
$$

\subsection{Measurements of Photosynthetic Parameters}

Leaf chlorophyll contents were measured using a SPAD-502plus meter (Konica Minolta, Tokyo, Japan). Leaf stomatal conductance was measured by an SC-1 leaf porometer (Decagon Devices, Pullman, WA, USA) $5 \mathrm{~h}$ after the onset of the light regime. $\mathrm{CO}_{2}$ assimilation and chlorophyll fluorescence were measured in the third true leaves using an open gas exchange system LI6400XT photosynthesis meter (LI-COR Biosciences, Lincoln, NE, USA). A $2 \mathrm{~cm}$ radius IRGA gas chamber was used for all the measurements, with the chamber temperature set at $25{ }^{\circ} \mathrm{C}, \mathrm{CO}_{2}$ flow rate at $400 \mu \mathrm{mol} \mathrm{mol}{ }^{-1}$, light intensity at $1,000 \mu \mathrm{mol}$ photons $\mathrm{m}^{-2} \mathrm{~s}^{-1}$, and relative humidity at $50 \% . \mathrm{CO}_{2}$ assimilation was measured $3 \mathrm{~h}$ after the onset of the light regime, while the chlorophyll fluorescence of dark-adapted leaves was measured early morning, before the onset of the light regime, after the plants were kept in darkness overnight.

\subsection{APX Enzyme Assay}

Crude leaf extracts were prepared essentially as described (Nanasato et al., 2010), with the following minor modifications: approximately $200 \mathrm{mg}$ of leaf tissues were ground to a fine powder using a pestle and mortar, with the aid of liquid nitrogen, in $1 \mathrm{ml}$ of homogenization buffer containing $50 \mathrm{mM}$ potassium phosphate, $\mathrm{pH} 7.0$, $1 \mathrm{mM}$ EDTA, $1 \mathrm{mM}$ sodium ascorbate, $1 \%$ (w/v) 3-[(3-cholamidopropyl) dimethyl-ammonio]-1-propanesulfonate (CHAPS), and 2\% polyvinylpolypyrrolidone. The detergent CHAPS was included in the buffer to solubilize thylakoid-bound APX (Veljovic-Jovanovic et al., 2001). The homogenized samples were centrifuged at $12,000 \times g$ for $20 \mathrm{~min}$ at $4{ }^{\circ} \mathrm{C}$ and then the supernatant was collected in a new tube. The extracts were desalted by running them through an Amicon Ultracel $3 \mathrm{~K}$ filter (Merck 
Millipore, Burlington, MA, USA), and their protein concentration was quantified using Protein Assay CBB Solution (Nacalai, Kyoto, Japan) and the Multiskan FC plate reader (Thermo Fisher Scientific, Waltham, MA, USA), using bovine serum albumin as the standard.

APX enzyme activity was measured essentially as described (Amako et al., 1994; Nanasato et al., 2010), with the following modifications: the reaction was performed in $1 \mathrm{ml}$ of assaying buffer containing $50 \mathrm{mM}$ potassium phosphate buffer, $\mathrm{pH} 7.0,1 \mathrm{mM}$ sodium ascorbate, $10 \mu \mathrm{l}$ of the crude leaf extract, and $1 \mathrm{mM}$ of $\mathrm{H}_{2} \mathrm{O}_{2}$. The assay was started by the addition of $\mathrm{H}_{2} \mathrm{O}_{2}$ substrate and a solution without the $\mathrm{H}_{2} \mathrm{O}_{2}$ substrate was used as a reference. The oxidation of ascorbate was continuously monitored by optical absorbance at $290 \mathrm{~nm}$ using a UH5300 spectrophotometer (Hitachi, Tokyo, Japan) and an absorption coefficient of $2.8 \times 10^{-3} \mathrm{M} \mathrm{cm}^{-1}$ (Nakano \& Asada, 1981) was used for the calculation of reaction rates. To separately quantify the two activities of chloroplast and cytosolic isoenzymes, the plant protein was incubated in the assay mixture with $10 \mu \mathrm{M}$ of $\mathrm{H}_{2} \mathrm{O}_{2}$ without ascorbate for $5 \mathrm{~min}$, and then residual activity was assayed as the cytosolic isoenzyme. The total APX was quantified without any prior incubation with $\mathrm{H}_{2} \mathrm{O}_{2}$ and used to calculate the value of chloroplast isoenzyme activity (Amako et al., 1994).

\subsection{Quantification of APX mRNA Expression}

Pairs of specific primers used for a RT-qPCR analysis of wild watermelon APX genes (Table A1) were designed using the Primer3 online tool (Untergasser et al., 2012). Total RNAs were extracted from the leaves of wild watermelon stressed for $0,3,5,7,9$, and 11 days, and cDNA synthesis were performed as described in Section 2.2. The mRNA abundance of the APX genes was measured by a Light-Cycler 480 (Roche Diagnostics, Mannheim, Germany), using a LightCycler 480 SYBR Green I Master Kit (Roche), according to the manufacturer's instruction. As reference genes, three sets of primers for $\gamma$-actin (ylsACT), $\alpha$-tubulin (ylsTUB), and glyceraldehyde-3-phosphate dehydrogenase (ylsGAPDH), which showed highly homogeneous expression in a wide range of tissue types, developmental stages and environmental stimuli in watermelon (Kong et al., 2014; Table S1), were used as controls, and their normalized value (Vandesompele et al., 2002) was used to calculate relative abundance of the APX mRNAs. The profiling of mRNA quantification was run with three biological replications, each consisting of an average of three technical replications.

\section{Results and Discussion}

\subsection{Structures of the Putative APX Genes in the Watermelon Genome}

Using the protein sequences deduced from all APX genes in Arabidopsis thaliana (Panchuk et al., 2002; Table A2) as the queries, we identified five homologous genes in the whole genome sequence of cultivar watermelon (Citrullus lanatus subsp. vulgaris cv. 97103) in the Cucurbit Genomics Database. These five putative APX genes were designated as CLAPX1 to CLAPX5, according to the order of highest sequence similarity with the translated sequence of Arabidopsis AtAPX1 (Table 1). These five genes were located on four different chromosomes, i.e., 1, 2, 3, and 8. Two genes for CLAPX1 and CLAPX3 on chromosome 2 were approximately $27 \mathrm{Mbp}$ apart, showing that the watermelon APX genes were not clustered but rather scattered in the genome. From the comparison between the genomic sequence and assembled EST sequences, or unigenes, 9-12 exons were predicted in these CLAPX genes (Figure 1).

Table 1. Summary on ascorbate peroxidase genes in watermelon

\begin{tabular}{|c|c|c|c|c|c|c|c|c|c|}
\hline \multirow[b]{2}{*}{ Isoenzyme name } & \multicolumn{4}{|c|}{ Cultivar watermelon (cv. 97103) } & \multicolumn{5}{|c|}{ Wild watermelon (Acc. No. 101117-1) } \\
\hline & Chr. $^{* 1}$ & $\begin{array}{l}\text { Locus } \\
\text { name }\end{array}$ & Locus position & Unigene ID & Acc. ID ${ }^{* 2}$ & $\begin{array}{l}\text { NT sim. } \\
(\%)^{* 3}\end{array}$ & $\begin{array}{l}\text { Length } \\
\text { of } \mathrm{AA}^{* 4}\end{array}$ & $\begin{array}{l}\text { WoLF } \\
\text { PSORT }^{* 5}\end{array}$ & Deep Loc ${ }^{*}$ \\
\hline CLAPX1 & 2 & $\mathrm{Cla} 013254$ & $30982510 . .30986181(-)$ & wmu3984 & MH178405 & 99.2 & 250 & chl & cyt \\
\hline CLAPX2 & 3 & Cla008291 & $2065040 . .2073452(-)$ & wmu 23766 & MH178406 & 99.2 & 249 & cyt & cyt \\
\hline CLAPX3 & 2 & Cla015833 & $3993709 . .3997744(+)$ & wmu34919 & MH178407 & 99.2 & 286 & cyt & per \\
\hline CLAPX4 & 1 & $\mathrm{Cla} 014301$ & $29858539 . .29862551(-)$ & wmu57455 & MH178408 & 99.5 & 296 & cyt & per \\
\hline CLAPX5-I & 8 & Cla013927 & $14858020 . .14864092(-)$ & wmu44297 & MH178409 & 99.1 & 427 & chl & $\mathrm{mit} / \mathrm{chl}$ \\
\hline CLAPX5-II & 8 & Cla013927 & $14858020 . .14864092(-)$ & wmu05603 & MH178410 & 99.0 & 378 & chl & $\mathrm{mit} / \mathrm{chl}$ \\
\hline
\end{tabular}

Note. ${ }^{* 1}$ Chromosome number in which APX gene was encoded. ${ }^{* 2}$ DDBJ/Genbank/EMBL accession numbers of APX sequences for the Acc. No. 101117-1. ${ }^{* 3}$ Percent similarity of CDS nucleotide sequences between APXs from cv. 97103 and Acc. No. 101117-1. ${ }^{* 4}$ Length of amino acid deduced from the CDSs in Acc. No. 101117-1 ${ }^{* 5}$ Subcellular location of the gene product in Acc. No. 101117-1 was predicted by WoLF-PSORT and DeepLoc-1.0 programs, and expressed as chl (chloroplast), cyt (cytosol), per (peroxisome), and mit (mitochondria). 
CLAPX5 was unique in that at least two mRNA variants with different splicing patterns were present; the one variant, designated CLAPX5-I, had a longer intron 11, at 290 nucleotides in length, whereas the other variant, designated CLAPX5-II, had a shorter intron 11, at 119 nucleotides in length, with concomitant increase in the length of exon 12. This observation suggests that alternative splicing generates two distinct mRNA transcripts from CLAPX5, as has been demonstrated in other plants (Mano et al., 1997; Yoshimura et al., 1999; Ishikawa \& Shigeoka, 2008).

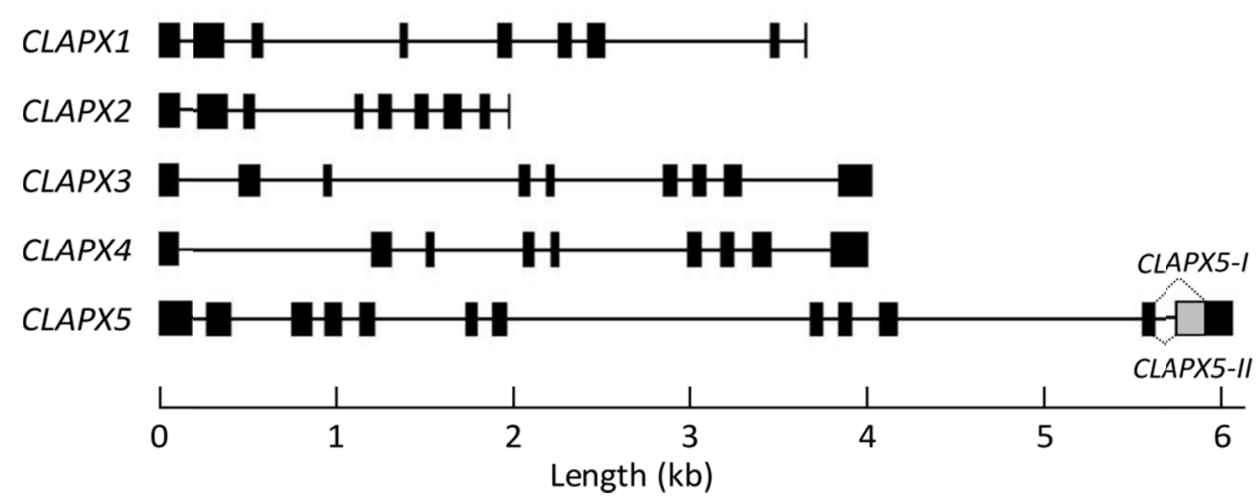

Figure 1. Schematic representation of watermelon APX gene structures

Note. Exon regions are shown as black boxes and intron regions are shown as horizontal lines. Patterns of alternative splicing in intron 11 of CLAPX5 are shown by broken triangular lines, and a region within exon 12 that is absent in one variant of the mature mRNA (CLAPX5-I), but present in another variant (CLAPX5-II), is shown as a gray box.

To examine the APX structure in wild watermelon, sets of PCR primers were designed in the 5'- and 3'-untranslated regions of APX gene, and corresponding full-length CDS fragments were amplified from cDNAs of wild watermelon. Consequently, a single PCR fragment was amplified for the genes CLAPX1, CLAPX2, $C L A P X 3$, and CLAPX4, while CLAPX5 showed two bands in the gel electrophoresis. Sequence analyses of the cloned fragments revealed highly conserved CDSs with nucleotide identity over $99 \%$ with the cv. 97103 counterparts, including the predicted two mRNA variants of CLAPX5-I and CLAPX5-II from the CLAPX5 gene (Table 1). Concerning CLAPX5, 3'-RACE experiments using a forward primer in exon 11 also detected two different splicing patterns, which matched with the results from the cDNA structural analyses (Figures A1, A2, and A3). In CLAPX5-II, an occurrence of the shorter intron 11 by the alternative splicing generated an in-frame stop codon immediately after exon 11, resulting in the shorter CDS of 1,134 nucleotides encoding a protein of 378 amino acid residues, in comparison to the CLAPX5-I variant of 1,281 nucleotides in length encoding a protein of 427 amino acid length (Table 1, Figures A1 and A2). The 3'-RACE experiments revealed that poly(A) tails began at two different cleavage sites (CS; Loke et al., 2005; Shen et al., 2008), designated proximal and distal CSs, which positioned at 47 and 75 nucleotides downstream, respectively, from the last nucleotide of the terminator codon of CLAPX-I (Figure A3). Both of the proximal and distal CSs occurred for CLAPX5-I and $C L A P X 5-I I$ transcripts, implying that the selection of CS for the poly(A) tail attachment may not influence the pattern of alternative splicing, in contrast to the case in spinach (Yoshimura et al., 1999). Putative near upstream elements (NUEs) of the cis-acting poly(A) signals were found at 22-28 nucleotides upstream of the CSs (Figure A3).

Deduced protein sequences of the six APX isoenzymes were in the range of 249-427 amino acids long and had mutually-conserved amino acid stretches (Figure 2). CLAPX5-I and CLAPX5-II are unique in that they had an extra sequence of 75 amino acids at their N-terminus, which were rich in serine residues. Prediction of intracellular localization of the protein products using the bioinformatics tools WoLF PSORT (Horton et al., 2007) and DeepLoc-1.0 (Armenteros et al., 2017) indicated that these N-terminal sequences may serve as chloroplast and mitochondrial/chloroplast transit peptides, respectively (Table 1). Although this inconsistent prediction awaits further experimental validations in future analyses, present observation suggests that CLAPX isoenzymes may distribute to different intracellular localizations.

Phylogenetic analysis of CLAPXS with Arabidopsis, spinach, and pumpkin APX isoenzymes demonstrated that $C L A P X 1$ and CLAPX2 were in the clade of cytosolic APXs from other plants, while CLAPX3 and CLAPX4 were 
more closely associated with microbody-type (Figure 3). CLAPX5-I and CLAPX5-II were rather distant from the cytosolic and microbody isoenzymes and strongly associated with chloroplast APXs from other plants. It is noteworthy that the C-terminal amino acid sequences of CLAPX5-I and CLAPX5-II had very strong sequence similarity with those of tAPX and sAPX from pumpkin (Figure A1 and A2 in Appendix). It has been shown that pumpkin tAPX and sAPX were generated from a single gene by alternative splicing, and the C-terminal extension in tAPX contains a putative thylakoid-spanning domain (Mano et al., 1997). Comparison of genomic sequences between CLAPX5 and pumpkin chloroplast APX showed that their C-terminus genomic regions were markedly conserved, including the conserved splicing junction between exon 11 and intron 11, and the alternative junctions between intron 11 and exon 12 (Figure A2). These observations suggest that the two mRNA variants from CLAPX5, i.e., CLAPX5-I and CLAPX5-II, may correspond to tAPX and sAPX, respectively, and the two watermelon APX isoenzymes may be generated from a single gene by an alternative splicing mechanism which is similar to that in pumpkin.

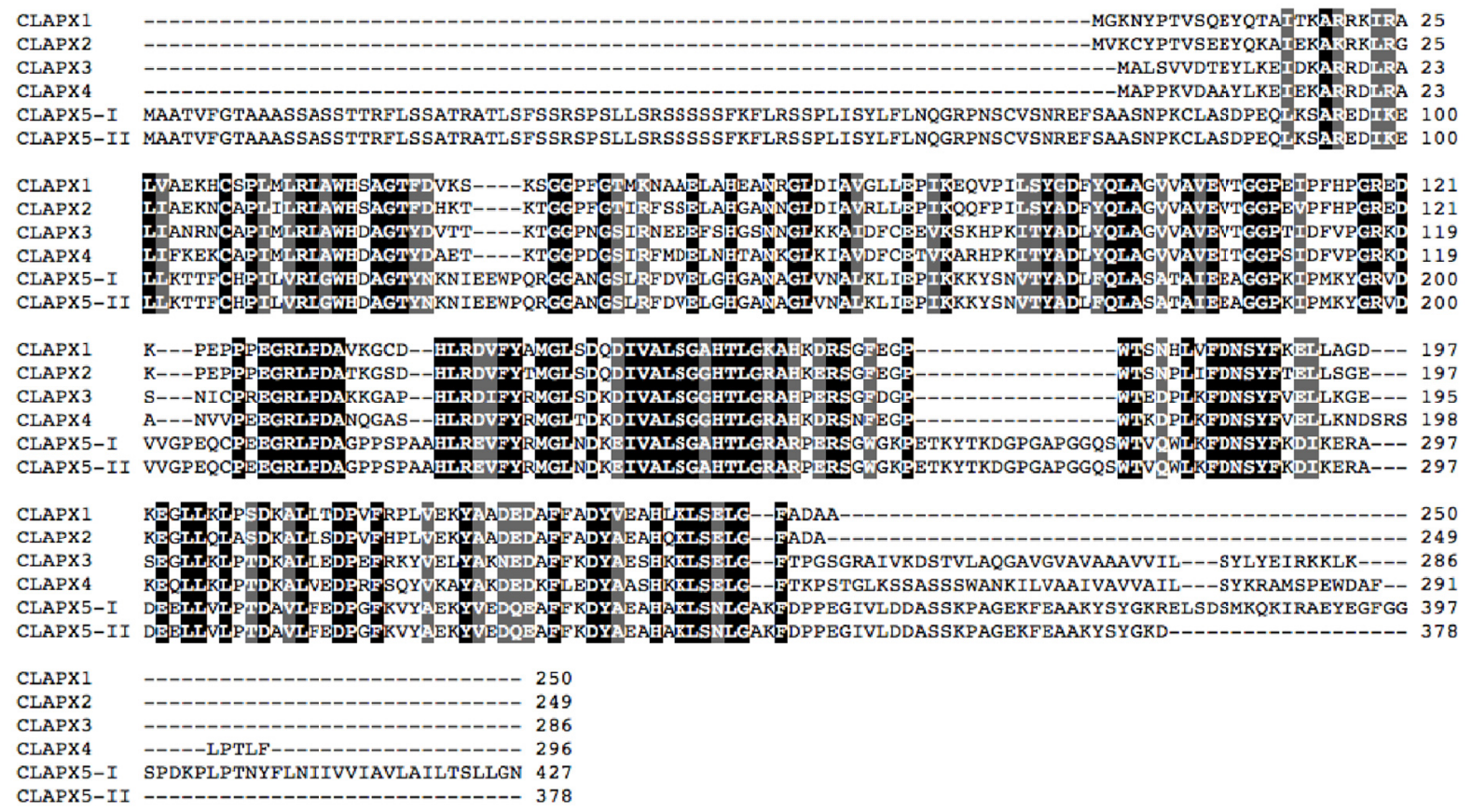

Figure 2. Amino acid sequence alignment of deduced wild watermelon APX isoenzymes

Note. Sequence alignment was performed by the ClustalW program. Completely conserved regions among the sequences are highlighted in black and highly similar regions are shown in gray. 


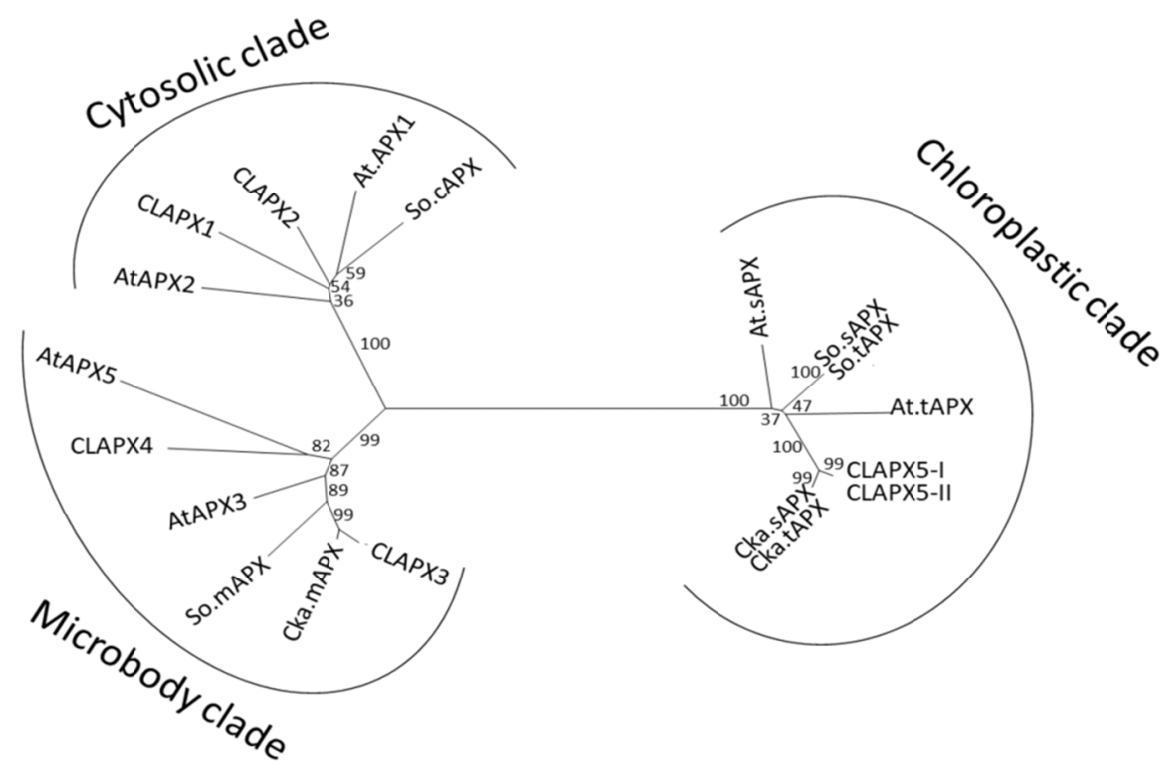

Figure 3. Phylogenetic analyses of CLAPX isoenzymes

Note. APX protein sequences from wild watermelon, Arabidopsis, spinach, and pumpkin were used to construct a phylogenetic tree using neighbor-joining algorithm in the ClustalW program. Sequence information and intracellular localizations of Arabidopsis (AtAPX1 to AtAPX5), spinach (So.sAPX and So.tAPX), and pumpkin APXs (Cka.sAPX, Cka.tAPX, and Cka.mAPX) are described elsewhere (Ishikawa \& Shigeoka 2008).

\subsection{Physiology of Wild Watermelon Under Drought}

Wild watermelon plants were grown until their fifth true leaves were fully expanded and drought stress was imposed by withholding irrigation. Soil moisture content was recorded at $154 \%$ at day 0 , gradually declined during drought, and dropped to $12 \%$ at day 11 of the drought period (Figure $4 \mathrm{a}$ ). Stomatal conductance at day 0 was $592.7 \pm 61.91 \mathrm{mmol} \mathrm{m}^{-2} \mathrm{~s}^{-1}$ and gradually decreased to $53.8 \pm 1.68 \mathrm{mmol} \mathrm{m}^{-2} \mathrm{~s}^{-1}$ at day 11 (Figure $4 \mathrm{~b}$ ). In contrast, leaf relative water content remained high at all time points during the experimental period (Figure 4c), remaining above $75 \%$, even when soil moisture dropped to $12 \%$. These features are consistent with previous observations (Nanasato et al., 2010; Sanda et al., 2011).

Measurements of SPAD value, an indicator of leaf chlorophyll level (Uddling et al., 2007), showed a value of $42.5 \pm 1.81$ at day 0 , then a slight decline was observed at days 3 and 5 after the drought onset, and dropped to the lowest value of $28.36 \pm 1.09$ recorded at day 11 (Figure 4d). These observations suggest that wild watermelon modestly reduced its photosynthetic potential during the drought period. 

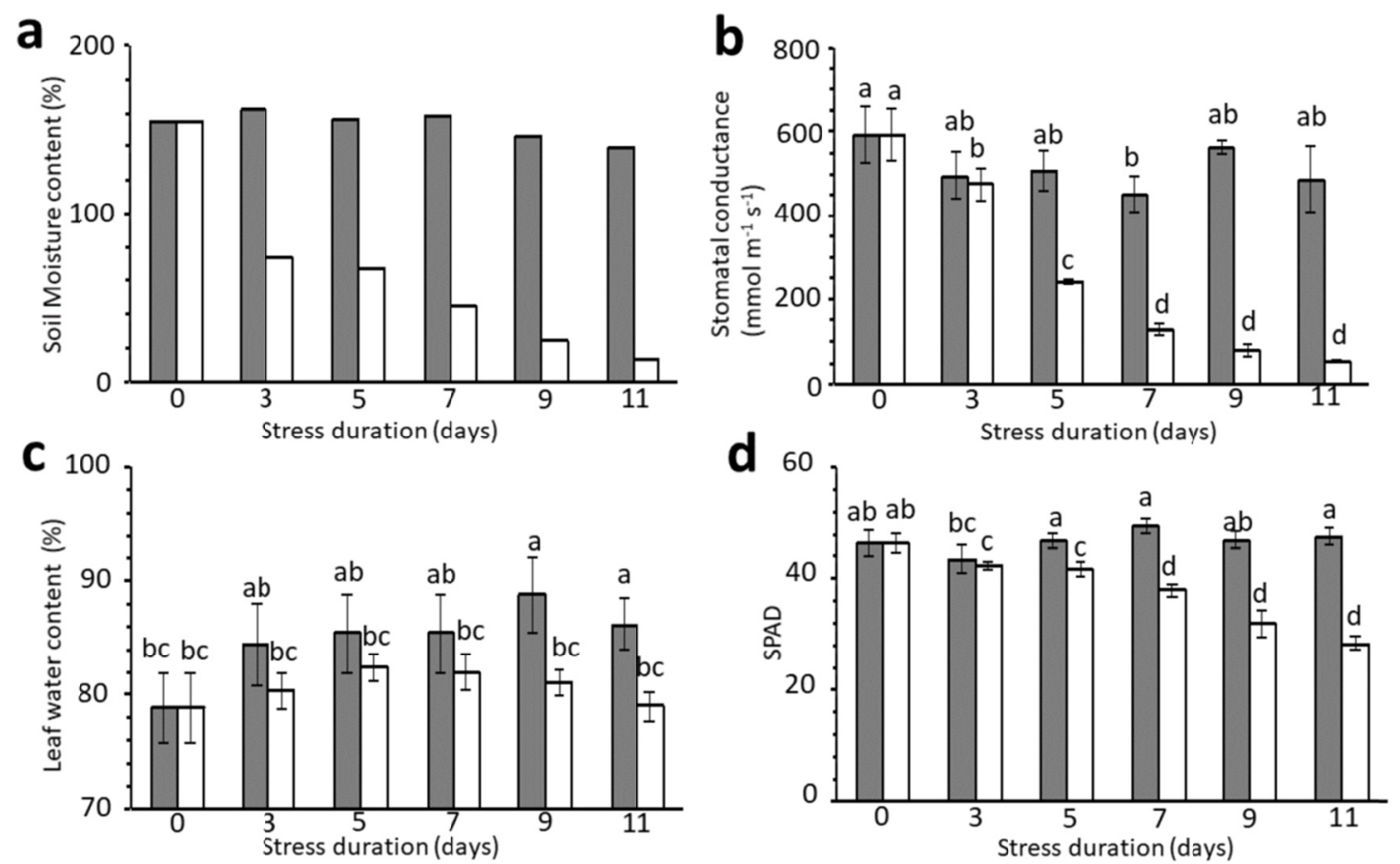

Figure 4. Changes in the water relation and foliar chlorophyll levels in wild watermelon under drought

Note. Changes in the soil moisture content (a), stomatal conductance (b), leaf water content (c), and SPAD value (d) during drought are presented for drought-stressed (open bars) and irrigated control (gray bars) conditions. In (a), the values are the result of a single measurement, whereas the values in (b), (c), and (d) are given as mean \pm SD $(n=3)$. Duncan multiple range tests were performed at $95 \%$ significance levels, and the significant differences among the values are indicated by the relevant letter(s) on top of each plot.

Photosynthetic $\mathrm{CO}_{2}$ assimilation at day 0 was $25.84 \pm 1.24 \mu \mathrm{mol} \mathrm{CO} \mathrm{Cm}^{-2} \mathrm{~s}^{-1}$, with a sudden drop of the values to $15.38 \pm 2.29$ and $8.89 \pm 4.23 \mu \mathrm{mol} \mathrm{CO} \mathrm{CO}_{2}^{-2} \mathrm{~s}^{-1}$ being observed at days 3 and 5, respectively, after the stress (Figure 5a). On day 11 of the stress, a minimal assimilation rate of $0.69 \pm 0.34 \mu \mathrm{mol} \mathrm{CO} \mathrm{Cm}^{-2} \mathrm{~s}^{-1}$ was recorded. Photochemical quenching (qP) of chlorophyll fluorescence, an indication of the proportion of open PSII reaction centers and representing a fraction of the light energy transmitted to the photochemical reaction, was $0.75 \pm 0.04$ at day 0 , and showed a steady decrease during drought, dropping to $0.14 \pm 0.02$ at day 11 (Figure $5 \mathrm{~b}$ ). The maximum PSII activity, $\mathrm{F}_{\mathrm{v}} / \mathrm{F}_{\mathrm{m}}$, an indicator of photoinhibition at photosystem II (Maxwell \& Johnson, 2000), was recorded as $0.72 \pm 0.05$ at day 0 (Figure $5 \mathrm{c}$ ), modestly decreased to $0.55 \pm 0.03$ at day 5 , and remained essentially unchanged thereafter. This observation indicated photoinhibitory damage, suggesting that the drought stress imposed on wild watermelon in this study might be more severe than in previous studies (e.g., Sanda et al., 2011), where the $F_{v} / F_{m}$ values remained unchanged during the study period.

A pronounced increase of the value for non-photochemical quenching (NPQ), a process where excess energy is dissipated as heat, was observed during drought (Figure 5d). The value reached a maximum of $0.81 \pm 0.03$ at day 11 of the stress. The induction of NPQ was consistent with a previous study by Sanda et al. (2011). 

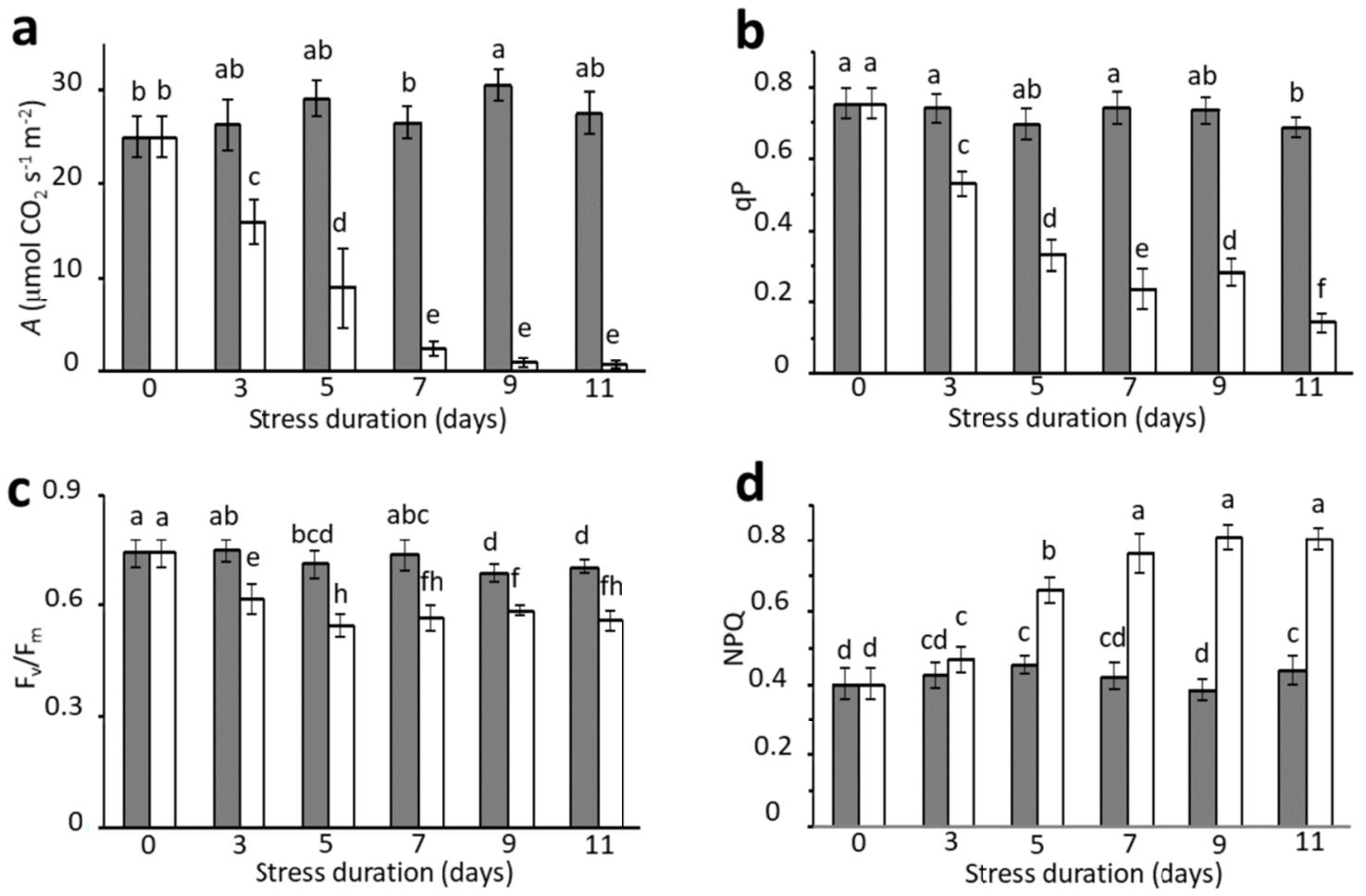

Figure 5. Photosynthesis and chlorophyll fluorescence parameters of the wild watermelon under drought

Note. Changes in the $\mathrm{CO}_{2}$ assimilation (a), qP value for photochemical quenching (b), $\mathrm{F}_{\mathrm{v}} / \mathrm{F}_{\mathrm{m}}$ for maximum PSII activity (c), and NPQ value for non-photochemical quenching (d) during drought (open bars) and irrigated control (gray bars) conditions are shown. The values are given as mean $\pm \mathrm{SD}(\mathrm{n}=3)$. Duncan multiple range tests were performed at $95 \%$ significance levels, and the significant differences among the values are indicated by the relevant letter(s) on top of each plot.

\subsection{Changes in the APX Enzyme Activity in the Leaves}

APX enzyme activities were measured using leaf crude extract. In this assay, chloroplast and cytosolic APX activities were measured separately on the basis of differential sensitivity to $\mathrm{H}_{2} \mathrm{O}_{2}$ in the absence of ascorbate (Kitajima, 2008). Consequently, a marked increase in chloroplast APX activity was observed during drought stress (Figure 6). The chloroplast enzyme activity at day 0 was $0.67 \pm 0.14 \mathrm{mmol} \mathrm{mg} \mathrm{protein}{ }^{-1} \mathrm{~min}^{-1}$, sharply increasing at day 3 and reaching its peak of $5.31 \pm 0.98 \mathrm{mmol} \mathrm{mg} \mathrm{protein}{ }^{-1} \mathrm{~min}^{-1}$ at day 5, showing a 7.9-fold increase in the enzyme activity. Then, the chloroplast activity slowly declined to the value of $2.91 \pm 0.21 \mathrm{mmol}$ $\mathrm{mg}$ protein ${ }^{-1} \mathrm{~min}^{-1}$ at day 11 . These observations showed a similar trend to, but different magnitude of increase from, a previous study by Nanasato et al. (2010), in which there was a 1.6-fold increase in the chloroplast activity. An increase was also observed for cytosolic APX activity, which was $0.57 \pm 0.08 \mathrm{mmol} \mathrm{mg} \mathrm{protein}^{-1}$ $\min ^{-1}$ at day 0 and increased to a peak value of $1.67 \pm 0.23 \mathrm{mmol} \mathrm{mg} \mathrm{protein}{ }^{-1} \mathrm{~min}^{-1}$ at day 5 , showing a 2.9-fold increase during drought. The behavior of cytosolic APX in this study was different from that observed in the study by Nanasato et al. (2010), in which no significant change in the cytosolic activity was observed. Reasons for the differential behaviors of chloroplast and cytosolic APX activities between the two studies are currently unclear, but it is possible that the stronger photo-inhibitory condition in the present study, as revealed by the lower $\mathrm{F}_{\mathrm{v}} / \mathrm{F}_{\mathrm{m}}$ value of 0.55 at day 5 , resulted in the stronger induction of APX enzymes in wild watermelon. Nevertheless, the present study confirms that chloroplast APX activity is significantly fortified during drought and high light stress conditions. 


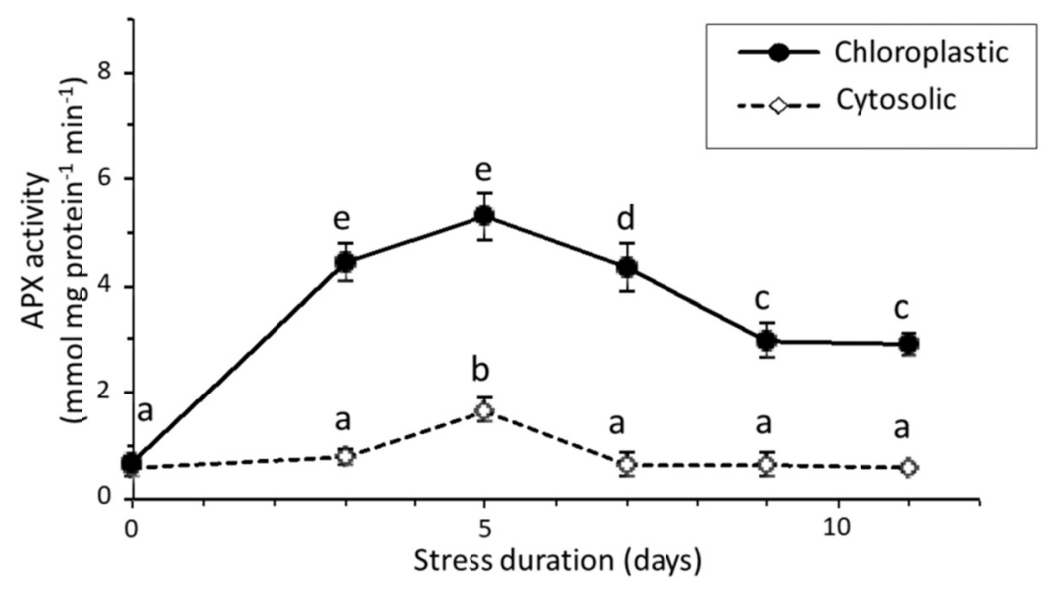

Figure 6. Changes in the APX enzyme activity in the leaves of wild watermelon under drought

Note. Solid lines with closed black circles represent the values for chloroplast APX specific activity, while dotted lines with white diamond symbols represent those of cytosolic APX specific activity. The values are given as mean \pm SD $(n=3)$. Duncan multiple range tests were performed at $95 \%$ significance levels, and the significant differences among the values are indicated by the relevant letter(s) on top of each symbol.

\subsection{Expression of the APX Gene Family in Wild Watermelon Leaves During Drought Stress}

Changes in the mRNA abundance for the six APX isoenzymes during drought were examined by RT-qPCR analyses. Concerning the CLAPX5 mRNAs that had two splicing variants as described above, primers were designed to span the exon-intron junction so as to detect each variant independently. Consequently, a complex and distinct profile of mRNA abundance was observed among the different APX genes (Figure 7). CLAPXI showed a sharp increase at day 3 and peaked at day 7 with a 3.3 -fold increase in the expression. After day 7 , the expression gradually returned to a level similar to that which preceded stress induction. The expression of CLAPX2 and CLAPX3 showed a slight increase during the early days of stress (days 3 and 5), and then a reduction for the remainder of the experiment. The expression pattern of CLAPX4 was complex in that a suppressed expression was observed at day 3 , and then gradually increased from day 5 , reaching its highest value at day 11.

Gene expression of CLAPX5-I variant showed an increase in the early phase of the stress at day 3, peaked at day 5, then decreased toward day 11 (Figure 7). The expression of CLAPX5-II showed a lower magnitude of increase at day 3, and stayed at the same level until day 9. It is noteworthy that the temporal induction of mRNA abundance for CLAPX5-I, which was most related to thylakoid APX in other plants (Figures 3, A1, and A2), resembled the temporal induction of chloroplast APX enzyme activity (Figure 6). This observation imply that transcriptional upregulation of CLAPX5-I in the early phase of drought stress may result in the fortification of chloroplast APX activity. Overall, these observations indicate the presence of distinct regulation for controlling mRNA abundance of the APX genes, which may reflect different physiological roles among APX isoenzymes in wild watermelon under drought. Future studies on the potential influence of this regulatory mechanism, to the intracellular redox status and physiological behavior in wild watermelon under drought, are anticipated. 


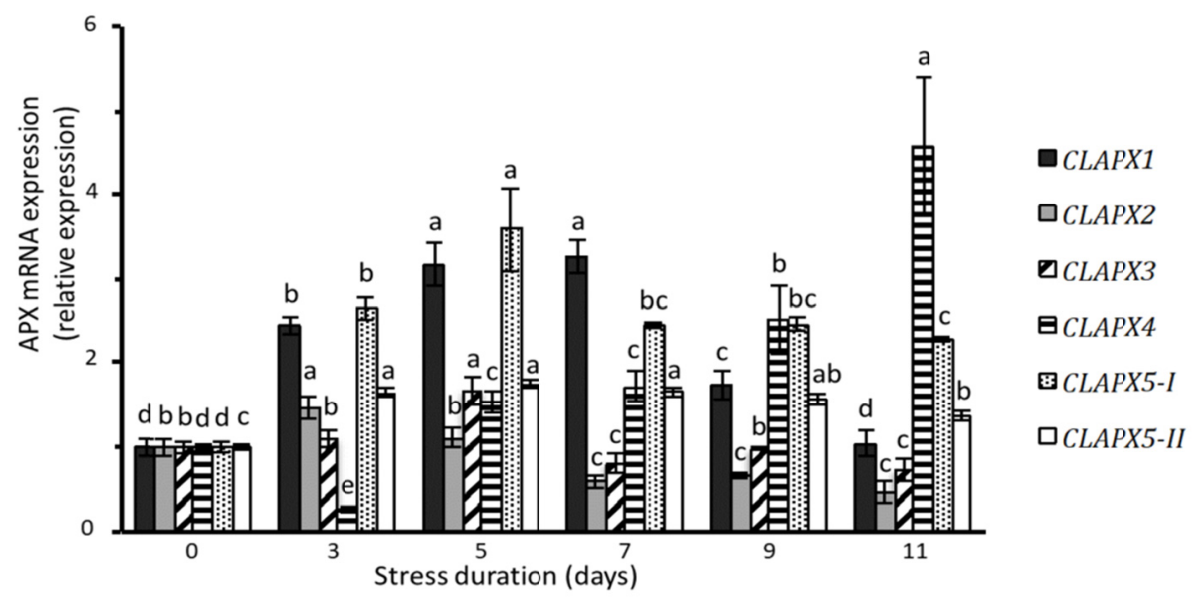

Figure 7. The mRNA expression profiles of CLAPX genes under drought

Note. Change in the mRNA abundance for CLAPX genes were examined by RT-qPCR analyses. Values are normalized by an average of three reference housekeeping genes for $\gamma$-actin, $\alpha$-tubulin, and GAPDH, and expressed as relative values to those of day 0 . The values are given as mean $\pm \operatorname{SD}(n=3)$. Duncan multiple range tests were performed at $95 \%$ significance levels, and the significant differences among the values are indicated by the relevant letter(s) on top of each bar.

\section{Conclusion}

In this study, structures of the APX gene family and their cDNAs in drought-tolerant wild watermelon were characterized. An alternative splicing event was found in one of the genes, CLAPX5, which potentially generated two different isoenzymes with different C-terminal amino acid sequences. Physiological and biochemical analyses of wild watermelon showed a reduction of stomatal conductance and $\mathrm{CO}_{2}$ assimilation rate, and an increase of NPQ, which was accompanied with a significant increase in the enzyme activities of both stromal and cytosolic APXs. Temporal patterns of mRNA abundance during drought were markedly different among the APX genes, suggesting that the respective APX isoenzymes may have different physiological roles in the adaptation of this plant to drought stress.

\section{Acknowledgements}

We thank Atsushi Kato for helping with seed preparation and various experimental procedures of wild watermelon. DNA sequencing was conducted by the Research Center for Bioscience and Technology, Tottori University. The authors gratefully acknowledge fundings for this co-research project from the Grant-in-Aid for Scientific Research (17K07755) from JSPS, and from the Joint Research Program and the Project Marginal Region Agriculture, the Arid Land Research Center, Tottori University, and the IPDRE Program, Tottori University.

\section{References}

Akashi, K., Yoshimura, K., Nanasato, Y., Takahara, K., Munekage, Y., \& Yokota, A. (2008). Wild plant resources for studying molecular mechanisms of drought/strong light stress tolerance. Plant Biotechnology, 25(3), 257-263. https://doi.org/10.5511/plant biotechnology.25.257

Amako, K., Chen, G. X., \& Asada, K. (1994). Separate assays specific for ascorbate peroxidase and guaiacol peroxidase and for the chloroplastic and cytosolic isozymes of ascorbate peroxidase in plants. Plant \& Cell Physiology, 35(3), 497-504. https://doi.org/10.1093/oxfordjournals.pcp.a078621

Armenteros, J. J. A., Sønderby, C. K., Sønderby, S. K., Nielsen, H., \& Winther, O. (2017). DeepLoc: prediction of protein subcellular localization using deep learning. Bioinformatics, 33(21), 3387-3395. https://doi.org/ 10.1093/bioinformatics/btx431

Asada, K. (2006). Production and scavenging of reactive oxygen species in chloroplasts and their functions. Plant Physiology, 141, 391-396. https://doi.org/10.1104/pp.106.082040

Barrs, H. D. (1968). Determination of water deficits in plant tissues. In T. T. Kozlowski (Ed.), Water Deficits and Plant Growth (Vol. 1, pp. 235-368). Academic Press, New York. 
Brozynska, M., Furtado, A., \& Henry, R. J. (2016). Genomics of crop wild relatives: expanding the gene pool for crop improvement. Plant Biotechnology Journal, 14(4), 1070-1085. https://doi.org/10.1111/pbi.12454

Gill, S. S., \& Tuteja, N. (2010). Reactive oxygen species and antioxidant machinery in abiotic stress tolerance in crop plants. Plant Physiology and Biochemistry, 48(12), 909-930. https://doi.org/10.1016/j.plaphy.2010. 08.016

Guo, S., Zhang, J., Sun, H., Salse, J., Lucas, W. J., Zhang, H., ... Xu, Y. (2013). The draft genome of watermelon (Citrullus lanatus) and resequencing of 20 diverse accessions. Nature Genetics, 45(1), 51-58. https://doi.org/10.1038/ng.2470

Hawkes, J. G. (1991). The importance of genetic resources in plant breeding. Biological Journal of the Linnean Society, 43(1), 3-10. https://doi.org/10.1111/j.1095-8312.1991.tb00578.x

Henry, R. J., \& Nevo, E. (2014). Exploring natural selection to guide breeding for agriculture. Plant Biotechnology Journal, 12(6), 655-662. https://doi.org/10.1111/pbi.12215

Hiner, A. N. P., Rodriguez-Lopez, J. N., Arnao, M. B., Raven, E. L., Novas, F. G., \& Acousta, M. (2000). Kinetic study of the inactivation of ascorbate peroxidase by hydrogen peroxide. Biochemical Journal, 348(2), 321-328. https://doi.org/10.1042/bj3480321

Horton, P., Park, K.-J., Obayashi, T., Fujita, N., Harada, H., Adams-Collier, C. J., \& Nakai, K. (2007). WoLF PSORT: protein localization predictor. Nucleic Acids Research, 35(Web Server Issue), W585-W587. https://doi.org/10.1093/nar/gkm259

Hu, B., Jin, J., Guo, A., Zhang, H., Luo, J., \& Gao, G. (2015). GSDS 2.0: An upgraded gene feature visualization server. Bioinformatics, 31(8), 1296-1297. https://doi.org/10.1093/bioinformatics/btu817

Ishikawa, T., \& Shigeoka, S. (2008). Recent advances in ascorbate biosynthesis and the physiological significance of ascorbate peroxidase in photosynthesizing organisms. Bioscience, Biotechnology, \& Biochemistry, 72(5), 1143-1154. https://doi.org/10.1271/bbb.80062

Kanehisa, K., Goto, S., Kawashima, S., \& Nakaya, A. (2002). The KEGG databases at GenomeNet. Nucleic Acids Research, 30(1), 42-46. https://doi.org/10.1093/nar/30.1.42

Karpinski, S., Escobar, C., Karpiska, B., Creissen, G., \& Mullineaux, P. M. (1997). Photosynthetic electron transport regulates the expression of cytosolic ascorbate peroxidase genes in Arabidopsis during excess light stress. Plant Cell, 9(4), 627-640. https://doi.org/10.1105/tpc.9.4.627

Kitajima, S. (2008). Hydrogen peroxide-mediated inactivation of two chloroplastic peroxidases, ascorbate peroxidase and 2-Cys peroxiredoxin. Photochemistry and Photobiology, 84(6), 1404-1409. https://doi.org/ 10.1111/j.1751-1097.2008.00452.x

Kitajima, S., Tomizawa, K., Shigeoka, S., \& Yokoya, A. (2006). An inserted loop region of stromal ascorbate peroxidase is involved in its hydrogen peroxide-mediated inactivation. FEBS Journal, 273(12), 2704-2710. https://doi.org/10.1111/j.1742-4658.2006.05286.x

Kong, Q., Yuan, J., Gao, L., Zhao, S., Jiang, W., Huang, Y., \& Bie, Z. (2014). Identification of suitable reference genes for gene expression normalization in qRT-PCR analysis in watermelon. PLoS ONE, 9, e90612. https://doi.org/10.1371/journal.pone.0090612

Li, G., Peng, X., Wei, L., \& Kang, G. (2013). Salicylic acid increases the contents of glutathione and ascorbate and temporally regulates the related gene expression in salt-stressed wheat seedlings. Gene, 529(2), 321-325. https://doi.org/10.1016/j.gene.2013.07.093

Loke, J. C., Stahlberg, E. A., Strenski, D. G., Haas, B. J., Wood, P. C., \& Li, Q. Q. (2005). Compilation of mRNA polyadenylation signals in Arabidopsis revealed a new signal element and potential secondary structures. Plant Physiology, 138(3), 1457-1468. https://doi.org/10.1104/pp.105.060541

Mano, S., Yamaguchi, K., Hayashi, M., \& Nishimura, M. (1997). Stromal and thylakoid-bound ascorbate peroxidases are produced by alternative splicing in pumpkin. FEBS Letters, 413(1), 21-26. https://doi.org/ 10.1016/s0014-5793(97)00862-4

Maxwell, K., \& Johnson, G. N. (2000). Chlorophyll fluorescence-A practical guide. Journal of Experimental Botany, 51(345), 659-668. https://doi.org/10.1093/jexbot/51.345.659

Mittler, R., Vanderauwera, S., Gollery, M., \& van Breusegem, F. (2004). Reactive oxygen gene network of plants. Trends in Plant Science, 9(10), 490-498. https://doi.org/10.1016/j.tplants.2004.08.009 
Miyake, C., Shinzaki, Y., Nishioka, M., Horiguchi, S., \& Tomizawa, K. (2006). Photoinactivation of ascorbate peroxidase in isolated tobacco chloroplasts: Galdieria partita APX maintains the electron flux through the water-water cycle in transplastomic tobacco plants. Plant \& Cell Physiology, 47(2), 200-210. https://doi.org/10.1093/pcp/pci235

Nakano, Y., \& Asada, K. (1981). Hydrogen peroxide is scavenged by ascorbate-specific peroxidase in spinach chloroplasts. Plant \& Cell Physiology, 22(5), 867-880. https://doi.org/10.1093/oxfordjournals.pcp.a076232

Nanasato, Y., Miyake, C., Takahara, K., Kohzuma, K., Munekage, Y. N., Yokota, A., \& Akashi, K. (2010). Mechanisms of drought and high light stress tolerance studied in a xerophyte, Citrullus lanatus (wild watermelon). In C. A. Rebeiz, et al. (Eds.), The Chloroplast. Advances in Photosynthesis and Respiration (Vol. 31). Basics and Applications (pp. 363-377). Springer, Dordrecht, Netherlands. https://doi.org/10.1007/ 978-90-481-8531-3_23

Panchuk, I. I., Volkov, R. A., \& Schoffl, F. (2002). Heat stress and heat shock transcription factor-dependent expression and activity of ascorbate peroxidase in Arabidopsis. Plant Physiology, 129(2), 838-853. https://doi.org/10.1104/pp.001362

Reynolds, S. G. (1970). The gravimetric methods of soil moisture determination: Part 1: A study of equipment, and methodological problems. Journal of Hydrology, 11(3), 258-273. https://doi.org/10.1016/0022-1694 (70)90068-5

Sanda, S., Yoshida, K., Kuwano, M., Kawamura, T., Nakajima, M., Akashi, K., \& Yokota, A. (2011). Responses of the photosynthetic electron transport system to excess light energy caused by water deficit in wild watermelon. Physiologia Plantarum, 142(3), 247-264. https://doi.org/10.1111/j.1399-3054.2011.01473.x

Shen, Y., Ji, G., Haas, B. J., Wu, X., Zheng, J., Reese, G. J., \& Li, Q. Q. (2008). Genome level analysis of rice mRNA 3'-end processing signals and alternative polyadenylation. Nucleic Acids Research, 36(9), 3150-3161. https://doi.org/10.1093/nar/gkn158

Shigeoka, S., Ishikawa, T., Tamoi, M., Miyagawa, Y., Takeda, T., Yabuta, Y., \& Yoshimura, K. (2002). Regulation and function of ascorbate peroxidase isoenzymes. Journal of Experimental Botany, 53(372), 1305-1319. https://doi.org/10.1093/jexbot/53.372.1305

Shikanai, T., Takeda, T., Yamauchi, H., Sano, S., Tomizawa, K., Yokota, A., \& Shigeoka, S. (1998). Inhibition of ascorbate peroxidase under oxidative stress in tobacco having bacterial catalase in chloroplasts. FEBS Letters, 428(1-2), 47-51. https://doi.org/10.1016/s0014-5793(98)00483-9

Uddling, J., Gelang-Alfredsson, J., Piikki, K., \& Pleijel, H. (2007). Evaluating the relationship between leaf chlorophyll concentration and SPAD-502 chlorophyll meter readings. Photosynthetic Research, 91(1), 37-46. https://doi.org/10.1007/s11120-006-9077-5

Untergasser, A., Cutcutache, I., Koressaar, T., Ye, J., Faircloth, B. C., Remm, M., \& Rozen, S. G. (2012). Primer3-new capabilities and interfaces. Nucleic Acids Research, 40(15), e115. https://doi.org/10.1093/ nar/gks596

Vandesompele, J., de Preter, K., Pattyn, F., Poppe, B., Van Roy, N., De Paepe, A., \& Speleman, F. (2002). Accurate normalization of real-time quantitative RT-PCR data by geometric averaging of multiple internal control genes. Genome Biology, 3(7), 0034. https://doi.org/10.1186/gb-2002-3-7-research0034

Veljovic-Jovanovic, S. D., Pignocchi, C., Noctor, G., \& Foyer, C. H. (2001). Low ascorbic acid in the vtc-1 mutant of Arabidopsis is associated with decreased growth and intracellular redistribution of the antioxidant system. Plant Physiology, 127(2), 426-435. https://doi.org/10.1104/pp.010141

Yoshimura, K., Ishikawa, T., Nakamura, Y., Tamoi, M., Takeda, T., Tada, T., ... Shigeoka, S. (1998). Comparative study on recombinant chloroplastic and cytosolic ascorbate peroxidase isozymes of spinach. Archives of Biochemistry and Biophysics, 353(1), 55-63. https://doi.org/10.1006/abbi.1997.0612

Yoshimura, K., Masuda, A., Kuwano, M., Yokota, A., \& Akashi, K. (2008). Programmed proteome response for drought avoidance/tolerance in the root of a $\mathrm{C}_{3}$ xerophyte (wild watermelon) under water deficits. Plant $\&$ Cell Physiology, 49(2), 226-241. https://doi.org/10.1093/pcp/pcm180

Yoshimura, K., Yabuta, Y., Ishikawa, T., \& Shigeoka, S. (2000). Expression of spinach ascorbate peroxidase isoenzymes in response to oxidative stresses. Plant Physiology, 123(1), 223-233. https://doi.org/10.1104/ pp.123.1.223 
Yoshimura, K., Yabuta, Y., Tamoi, M., Ishikawa, T., \& Shigeoka, S. (1999). Alternative spliced mRNA variants of chloroplasts ascorbate peroxidase isoenzymes in spinach leaves. Biochemical Journal, 338(1), 41-48. https://doi.org/10.1042/0264-6021:3380041

You, J., \& Chan, Z. (2015). ROS regulation during abiotic stress responses in crop plants. Frontiers in Plant Science, 6, 1092. https://doi.org/10.3389/fpls.2015.01092

\section{Appendix}

Table A1. Primer sequences used in this study

\begin{tabular}{|c|c|c|}
\hline Usage & Primer name & Primer sequence \\
\hline \multicolumn{3}{|c|}{ Full length $c D N A$ amplification } \\
\hline & APX-1F & СТTTTCAAGAGAATCTCAGCC \\
\hline & APX-1R & CGTTTGAACTTCTGGAGAAG \\
\hline & APX-2F & САTTTTTTCCAAGTTTCATCACC \\
\hline & APX-2R & TTTTCCCCCGTTCCAATTGC \\
\hline & APX-3F & TGCTCTGTAGCCTCCTCCC \\
\hline & APX-3R & AATCGACCTTGCTACTGTAA \\
\hline & APX-4F & TCTGCATTCTTATCCAAGATTTCA \\
\hline & APX-4R & TGGAAGAATATCTTTCTTCTGTATAA \\
\hline & APX-5F & GAAAACTCAAATTTCAACTAAATCC \\
\hline & APX-5R & CTCATAAATAGATTAATTTAAACACTCAAA \\
\hline \multicolumn{3}{|r|}{$-\cdots$} \\
\hline & Apx $5-1086 \mathrm{~F}+\mathrm{Kpn}$ & GCGGTACCTGCTGGAGAGAAGTTCG \\
\hline & 3sites-Adaptor & CTGATCTAGAGGTACCGGATCC \\
\hline & 3sites-Poly-dT & CTGATCTAGAGGTACCGGATCCTTTTTTTTTTTTTTTT \\
\hline \multicolumn{3}{|c|}{$R T-q P C R$ for CLAPX } \\
\hline & CLAPX1-77F & TTGTTGCTGAGAAGCACTGC \\
\hline & CLAPX1-195R & TTCCGCTGCGTTCTTCATTG \\
\hline & CLAPX2-79F & ATCGCTGAGAAGAACTGTGC \\
\hline & CLAPX2-163R & CACCGGTCTTGGTTTTCTGGTC \\
\hline & CLAPX3-71F & TCATTGCCAATCGGAACTGC \\
\hline & CLAPX3-162R & AGGCCCACCAGTTTTTGTAG \\
\hline & CLAPX4-134F & ACGACGCTGAAACGAAAACC \\
\hline & CLAPX4-253R & TGGCCTTCACAGTTTCACAG \\
\hline & CLAPX5_I-1004F & CTGAAGCCCATGCCAAACTC \\
\hline & CLAPX5_I-144R & CTGATAGCTCTCTCTTTCCATATGAG \\
\hline & CLAPX5_II-1004F & CTGAAGCCCATGCCAAACTC \\
\hline & CLAPX5_II-1146R & ACTTGTTTTTTAATCCTTTCCATATGAGTA \\
\hline \multicolumn{3}{|c|}{$R T-q P C R$ for rerefence genes } \\
\hline & Actin-F & TGGTCGTACAACAGGTTGTGC \\
\hline & Actin-R & TTCGGCAGTGGTTGTGAACATG \\
\hline & Tubulin-F & GGTCAGGAAGTTGGCTGATAAC \\
\hline & Tubulin-R & CACTGACAAGCGCTCTAACAAC \\
\hline & GAPDH-F & CCGATGAGGATGTTGTTCTCTAC \\
\hline & GAPDH-R & CATTGTCGTACCAAGTCACCAG \\
\hline \multicolumn{3}{|c|}{ Sequencing } \\
\hline & M13-F & GTAAAACGACGGCCAG \\
\hline & M13-R & CAGGAAACAGCTATGAC \\
\hline
\end{tabular}


Table A2. Accession numbers for plant APX genes that were used in this study.

\begin{tabular}{lll}
\hline Organism & Gene & Accession number $^{* 1}$ \\
\hline Arabidopsis thaliana & AtAPX1 & AT1G07890.1 \\
& AtAPX2 & AT3G09640.1 \\
AtAPX3 & AT4G35000.1 \\
AtAPX5 & AT4G35970.1 \\
At.sAPX & AT1G77490.1 \\
At.tAPX & AT4G08390.1 \\
Spinach & So.cAPX & D85864 \\
& So.sAPX & D84104 \\
& So.tAPX & D83669 \\
Pumpkin & Cka.mAPX & D77997 \\
& Cka.sAPX & AB070626 \\
Cka.tAPX & D88420 \\
\hline
\end{tabular}

Note. ${ }^{* 1}$ For Arabidpsis, AGI locus identifiers were shown. For spinach and pumpkin, NCBI/EMBL/DDBJ accession numbers were shown.

\section{a}

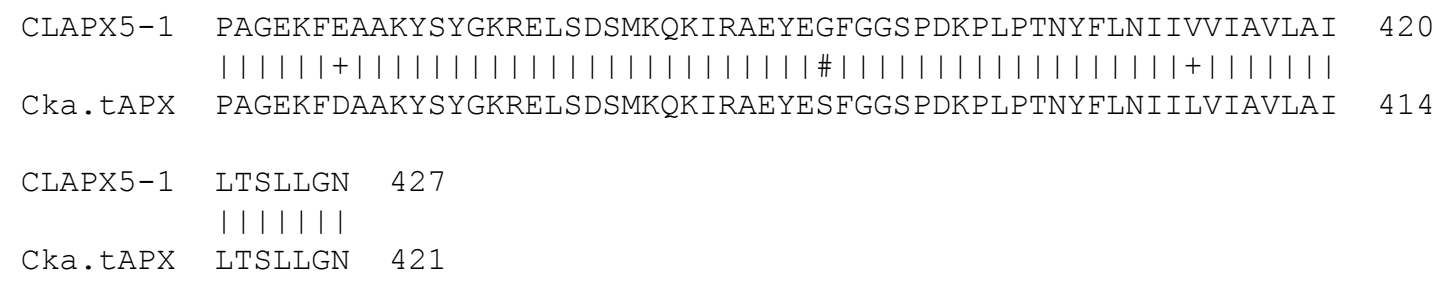

b

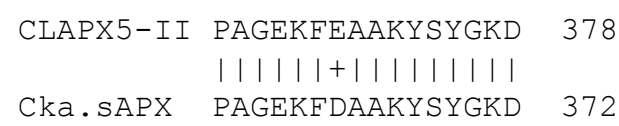

Figure A1. C-terminus amino acid alignment between CLAPX5 and pumpkin chloroplast APXs

Note. Amino acid alignments of C-terminal region for a pair of CLAPX5-I and pumpkin thylakoid Cka.tAPX (a), and a pair of CLAPX5-II and pumpkin stromal Cka.sAPX (b). The region spanning exon 11 and exon 12 is shown for each pair. Identical amino acids are labeled by vertical lines, similar amino acid residues between the pair are labeled as +, and amino acids with different chemical properties are labeled as \#. 
$\begin{array}{llllllllllllllll}P & A & G & E & K & F & E & A & A & K & Y & S & Y & G & K & \text { (i) }\end{array}$

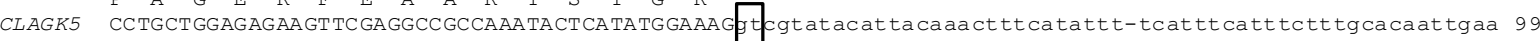

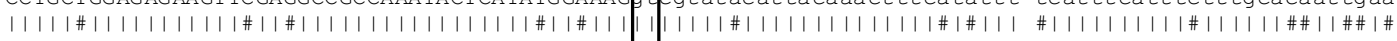
Cka. APX CCTGCAGGAGAGAAGTTTGATGCCGCCAAATACTCATACGGGAAgg cgtatncattacaacttct tntttcccatttcatttatttgcatgataat 100 $\begin{array}{llllllllllllllllllll}P & A & G & E & K & F & D & A & A & K & Y & S & Y & G & K\end{array}$

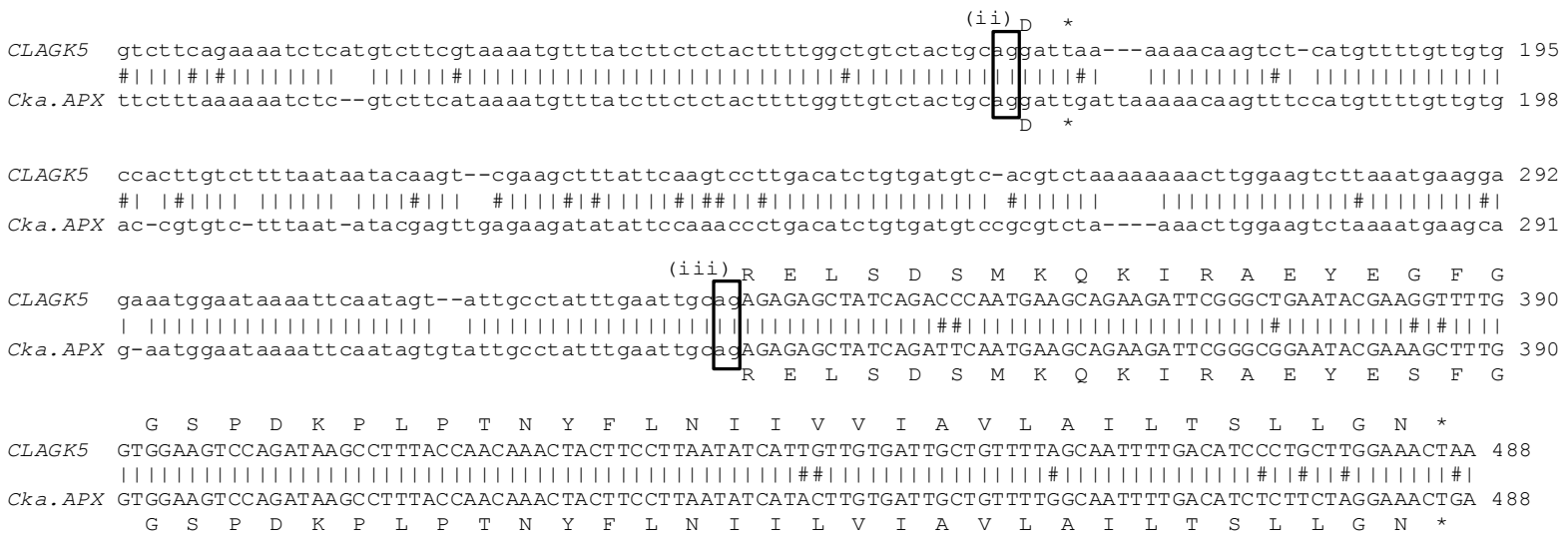

Figure A2. Comparison of genome sequence between CLAPX5 and the pumpkin chloroplast APX gene in the region that corresponds to the $\mathrm{C}$-terminus

Note. A nucleotide sequence alignment between CLAPX5 and the pumpkin chloroplast APX gene in their C-terminal region is shown. The region spanning exon 11 and exon 12 is presented. Identical nucleotides between the two sequences are labeled as vertical lines and different nucleotides are labeled as \#. Deduced amino acid sequences for CLAPX5 and pumpkin chloroplast APX are presented on top of and below the nucleotide sequences, respectively. Terminator codons are indicated by asterisk (*). A conserved "gt" motif at the beginning of intron 11 is boxed and labeled as (i), while the conserved "ag" motif for the termination of intron 11 for CLAPX5-II and CLAPX5-I are also boxed and labeled as (ii) and (iii), respectively.

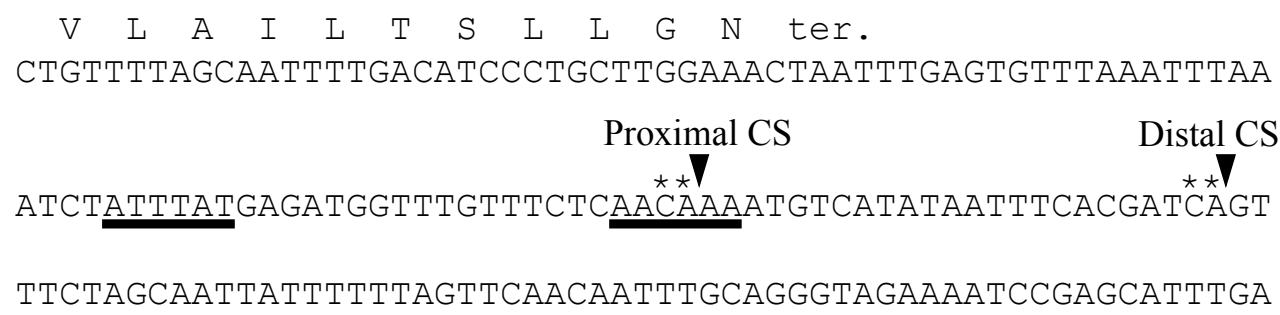

Figure A3. The observed cleavage sites for addition of poly(A) tails, and putative cis-acting poly(A) signals in the $C L A P X 5$ gene

Note. The positions of observed proximal and distal cleavage sites (CSs) for the addition of poly(A) tails were shown by the downward arrows, and the conserved CS motifs were indicated with asterisks on top of the dinucleotides. The hexanucleotides for the putative near upstream elements (NUEs) of the poly(A) signals (Loke et al., 2005) are shown by the underlines.

\section{Copyrights}

Copyright for this article is retained by the author (s), with first publication rights granted to the journal.

This is an open-access article distributed under the terms and conditions of the Creative Commons Attribution license (http://creativecommons.org/licenses/by/4.0/). 